\title{
Mild Systemic Hypothermic Circulatory Arrest Using a Frozen Elephant Trunk Graft with Endo-Balloon Occlusion for Total Arch Replacement
}

\author{
Yoshihiro Goto, MD, Soh Hosoba, MD, PhD, Yuichiro Fukumoto, MD, Sho Takagi, MD, PhD, \\ Junji Yanagisawa, MD
}

Department of Cardiovascular Surgery, Toyohashi Heart Center, Toyohashi, Japan

\section{ABSTRACT}

Background: Stroke and paraplegia are serious complications of total aortic arch replacement (TAR). Hypothermic circulatory arrest and cerebral perfusion reduce the risk of neurologic complications, but longer circulatory arrest time remains a risk factor for such complications. We utilized a frozen elephant trunk (FET) with endo-balloon occlusion under mild systemic hypothermia, which allowed us to shorten circulatory arrest time.

Methods: Between April 2007 and May 2020, 72 patients underwent elective TAR using antegrade cerebral perfusion (ACP). They were divided into 2 groups. 64 patients received conventional TAR with moderate systemic hypothermic (bladder temperature, $25-28^{\circ} \mathrm{C}$ ) circulatory arrest (group C). We used a FET with endo-balloon occlusion and retrograde perfusion through the femoral artery for the newest 8 patients who had mild hypothermic (bladder temperature of $30^{\circ} \mathrm{C}$ ) circulatory arrest (group B).

Results: The mean operation time $(257.5 \pm 42.1$ versus $327.8 \pm 84.9 \mathrm{~min}, P=.023)$, CPB time $(144.4 \pm 28.1$ versus $178.2 \pm 26.4 \mathrm{~min}, P=.003)$, cardiac arrest time $(75.5 \pm 21.2$ versus $95.7 \pm 56.4 \mathrm{~min}, P<.001)$, SCP time $(100.8 \pm 25.5$ versus $124 \pm 23.2 \mathrm{~min}, \mathrm{P}<.001)$, lower body circulation arrest time $(17.2 \pm 4.2$ versus $62.5 \pm 19.3 \mathrm{~min}, P<.001)$ were significantly shorter in the endo-balloon occlusion group. There were no perioperative neurological and renal complications or mortality in FET group. The new technique enabled a decrease in mechanical ventilation time $(8.6 \pm 1.4$ versus 13.9 $\pm 5.7 \mathrm{~min}, P=.015)$ and hospital length of stay $(9.7 \pm 1.8$ versus $18.3 \pm 4.6 \mathrm{~min}, P=.005$ ).

Conclusion: FET using an endo-balloon occlusion with mild hypothermia is a safe and an effective approach in TAR.

\section{INTRODUCTION}

Stroke and paralysis are serious complications of total arch replacement (TAR). Antegrade cerebral perfusion (ACP) and hypothermic systemic circulatory arrest are well-established

Received fune 9, 2020; accepted fuly 13, 2020.

Correspondence: Sob Hosoba, MD, PhD, Department of Cardiovascular Surgery, Toyohashi Heart Center, 21 Gobutori, Toyohashi, 4418530, Japan; +81 532-37-3377; fax: +81 532-37-3366 (e-mail: sob.hosoba@gmail.com). neuroprotective strategies [Kazui 2000]. However, the ideal temperature for systemic circulatory arrest remains controversial. Open stent graft technique, also known as frozen elephant trunk (FET), is a procedure in which a covered endovascular stent graft is deployed into the descending aorta [Kato 1996]. This approach, in combination with endoballoon occlusion and distal perfusion started from the femoral arterial cannula during distal anastomosis, can markedly shorten circulatory arrest time. Herein, we report our experience using an FET graft in combination with mild hypothermia for TAR.

\section{MATERIALS AND METHODS}

Between April 2007 and May 2020, 72 patients underwent elective TAR with ACP technique for thoracic aortic aneurysms at Toyohashi Heart Center. 72 patients who underwent TAR were categorized into two groups. 64 patients underwent conventional TAR with moderate hypothermic (bladder temperature, $25-28^{\circ} \mathrm{C}$ ) circulation arrest (group C). The newest 8 cases were treated using an FET graft with endoballoon occlusion under mild hypothermic (bladder temperature, $30^{\circ} \mathrm{C}$ ) circulation arrest (group B). Table 1 demonstrates the baseline conditions of these patients in each group. The

Table 1. Baseline Characteristics of Patients

\begin{tabular}{lccc}
\hline & Group B ( $\mathrm{n}=8)$ & Group C ( $\mathrm{n}=64)$ & $P$ \\
\hline Age, years & $69.1 \pm 12.4$ & $72.5 \pm 9.2$ & .42 \\
Body mass index, $\mathrm{kg} / \mathrm{m}^{2}$ & $20.8 \pm 2.6$ & $23.7 \pm 2.9$ & .148 \\
Male sex, $\mathrm{n}(\%)$ & $7(87.5)$ & $45(71.4)$ & .63 \\
Diabetes, $\mathrm{n}(\%)$ & $1(12.5)$ & $7(10.9)$ & .76 \\
Hypertension, $\mathrm{n}(\%)$ & $6(75)$ & $58(90)$ & .056 \\
Ejection fraction, \% & $61.1 \pm 8.1$ & $61.6 \pm 6.7$ & .83 \\
Hematocrit, \% & $38.6 \pm 5.2$ & $38.1 \pm 5.2$ & .78 \\
Renal insufficiency, $\mathrm{n}(\%)$ & 0 & $3(4.6)$ & .69 \\
Smoking history, $\mathrm{n}(\%)$ & $1(12.5)$ & $9(14)$ & .69 \\
Serum creatinine level, $\mathrm{mg} / \mathrm{dL}$ & $1.2 \pm 0.7$ & $1.2 \pm 1.1$ & .48 \\
History of cerebrovascular & $1(12.5)$ & $5(7.8)$ & .52 \\
disease, $\mathrm{n}$ (\%) & & &
\end{tabular}




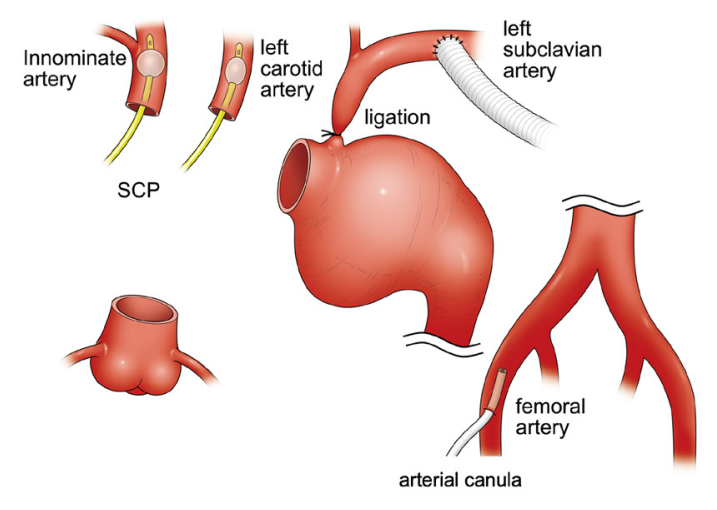

Figure 1. Arterial cannulation is achieved using the left subclavian artery using a $9 \mathrm{~mm}$ graft. $12 \mathrm{Fr}$ arterial cannula is inserted in the femoral artery. Antegrade selective cerebral perfusion is established through the brachiocephalic, left common carotid artery and left subclavian artery.

landing zone of the FET graft was determined to be above the level of aortic valve, with multi-dimensional computed tomography preoperatively.

The acute kidney injury (AKI) criteria and classification were those of the RIFLE [Uchino 2006]. The AKI criteria stipulate a percentage increase in serum creatinine of $50 \%$ or more.

\section{Surgical Metbod}

All operations were performed via median sternotomy. The arterial cannulation site was the ascending aorta; circulatory arrest was implemented at the bladder temperature of $25-28^{\circ} \mathrm{C}$ with ACP in conventional TAR. ACP was established through brachiocephalic and left common carotid arteries. Distal anastomosis to the quadrifurcated graft was created. After distal anastomosis, rewarming was started and proximal anastomosis was performed. The left subclavian, common carotid, and brachiocephalic artery were then reconstructed.

\section{Surgical Procedure for End-Balloon Occlusion Technique with FET}

The arterial cannula was placed in the left subclavian artery using a $9 \mathrm{~mm}$ graft, with the venous cannula inserted in the right atrium. Four Fr sheath and 12 Fr arterial cannula was inserted in bilateral femoral artery (Figure 1). Cooling was initiated at the time of cardiopulmonary bypass institution. Circulatory arrest was initiated when the bladder temperature reached $30^{\circ} \mathrm{C}$. ACP was established from the brachiocephalic and left common carotid arteries, with left subclavian artery ligation. A 15 Fr balloon-tipped cannula was inserted into the brachiocephalic artery and $12 \mathrm{Fr}$ cannula into the left common carotid, through which ACP flowed at $10 \mathrm{~mL} / \mathrm{kg} /$ min with the perfusate temperature of $25-28^{\circ} \mathrm{C}$. The aorta was transected between the left common carotid artery and left subclavian artery. Distal anastomosis was performed using FROZENIX (Japan Lifeline, Tokyo, Japan) FET graft. We
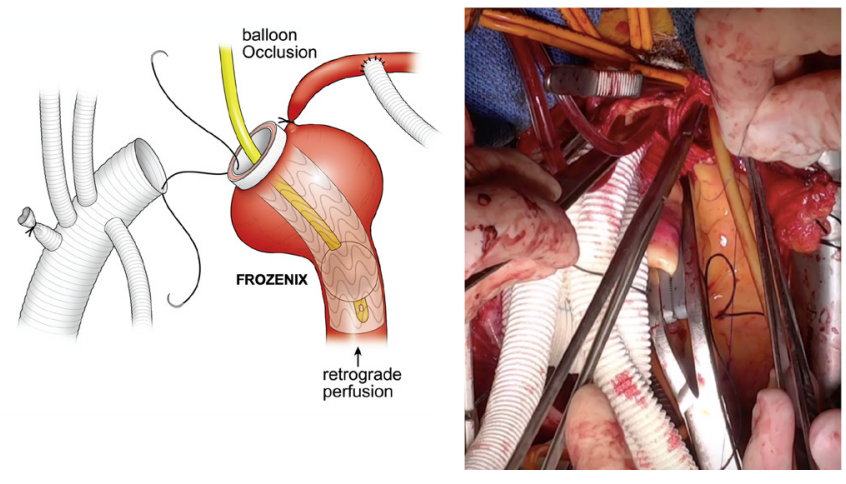

Figure 2. After frozen elephant trunk is deployed, retrograde lower body perfusion is initiated utilizing balloon-clamp during distal anastomosis.

inserted a guide wire from 4 Fr sheath at the femoral artery through to the FET graft lumen to guide the graft deployment in the descending aorta. After debris and air were flushed out with femoral perfusion, distal retrograde perfusion was established from the femoral artery by occluding the FET graft using a Foley catheter balloon (C. R. Bard, USA) (Figure 2). Lower body perfusion commenced at a flow rate of $2 \mathrm{~L} / \mathrm{min}$, adjusted to maintain a femoral artery pressure of 50-70 $\mathrm{mmHg}$. Distal anastomoses to the 4 branched J graft (Japan Lifeline, Tokyo, Japan) was performed by incorporating felt strips. After the distal anastomosis was completed, we initiated rewarming via systemic perfusion from a branch of the graft. During rewarming, we proceeded with completion of the proximal anastomosis and reconstruction of the left common carotid and brachiocephalic arteries. The left subclavian artery was reconstructed via the left infra-subclavian incision (Figure 3).

\section{Statistical Analysis}

Demographics of the patients and outcome variables were expressed either as a percentage of the total or as mean \pm standard deviation. Group comparisons were performed using a two-tailed Student $t$ test for continuous variables and the Pearson chi-square test for categorical variables. A $P$ value of less than .05 was considered to be statistically significant. All statistical analyses were performed using SPSS version 22 software (SPSS, Chicago, IL, USA).

\section{RESULTS}

Intraoperative and postoperative characteristics are shown in Table 2. The mean operation time $(257.5 \pm 42.1$ versus $327.8 \pm 84.9 \mathrm{~min}, P=.023), \mathrm{CPB}$ time $(144.4 \pm 28.1$ versus $178.2 \pm 26.4 \mathrm{~min}, P=.003)$, cardiac arrest time $(75.5 \pm 21.2$ versus $95.7 \pm 56.4 \mathrm{~min}, P<.001)$, SCP time $(100.8 \pm 25.5$ versus $124 \pm 23.2 \mathrm{~min}, P<.001)$, lower body circulation time $(17.2 \pm 4.2$ versus $62.5 \pm 19.3 \mathrm{~min}, P<.001)$ were remarkably shorter for the endo-balloon occlusion group, respectively.

The bladder temperatures at initiation $(30.1 \pm 0.7$ versus $26.5 \pm 0.9, P<.001)$ and lower bladder temperatures $(28.0 \pm$ 0.8 versus $24.9 \pm 0.7, P=.001)$ were significantly different. 

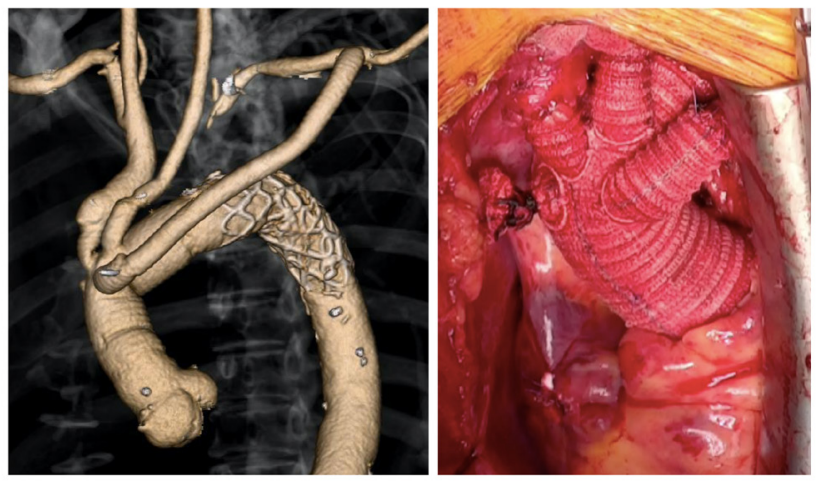

Figure 3. Reconstruction of the aorta. The left subclavian artery is reconstructed by anastomosing a $9 \mathrm{~mm}$ graft and the branch of the J graft through intracoastal space.

The incidence of postoperative AKI was at a lesser frequency compared with conventional methods (0 versus $12.5 \%, P=.37)$.

The patients who underwent the endo-balloon occlusion technique required shorter periods of postoperative mechanical ventilation $(8.6 \pm 1.4$ versus $13.9 \pm 5.7 \mathrm{~min}, P=.015)$, as well as of postoperative hospital stay $(9.7 \pm 1.8$ versus $18.3 \pm 4.6$ day, $P=.005)$ than patients who underwent conventional TAR.

All patients were alive at 30 days after the surgery without any neurological complications or any hemodialysis in the endo-balloon group.

The endo-balloon group had no endoleakage on postoperative computed tomography with contrast.

\section{DISCUSSION}

Our series demonstrated that FET in combination with endo-balloon occlusion and distal perfusion started from the femoral arterial cannula during distal anastomosis may shorten the hypothermic arrest time markedly with excellent clinical outcomes. Stroke and paraplegia are devastating complications of TAR. ACP and hypothermic systemic circulation arrest provide neurological protection during the distal anastomosis. However, hypothermia is accompanied by the risk of coagulopathy and organ dysfunction [Yau 1992; Preventza 2017]. Our approach to TAR has implications in further improving the outcomes of patients with complex aortic disease, since shortening the duration of hypothermia can minimize the risk of such adverse events.

The optimal temperature for hypothermia remains actively debated, and the safe limit of circulation arrest time under mild hypothermia for neurological and visceral organ protection is unknown. The lower body circulatory arrest temperature of $28^{\circ} \mathrm{C}$ has been proposed as a safe threshold. Zierer et al recommend that if the distal pathology is complex with an anticipated ACP time of $>60 \mathrm{~min}$, then the patient should be cooled down to $28^{\circ} \mathrm{C}$ for spinal cord protection [Zierer 2011].

Postoperative acute kidney injury (AKI) in patients undergoing TAR is also a common complication. The risk
Table 2. Intraoperative and Postoperative Characteristics of Patients

\begin{tabular}{lccc}
\hline & $\begin{array}{c}\text { Group B } \\
(\mathrm{n}=8)\end{array}$ & $\begin{array}{c}\text { Group C } \\
(\mathrm{n}=64)\end{array}$ & $\mathrm{P}$ \\
\hline Operation time, min & $257.5 \pm 42.1$ & $327.8 \pm 84.9$ & .023 \\
CPB time, min & $144.4 \pm 28.1$ & $178.2 \pm 26.4$ & .003 \\
Cardiac arrest time, min & $75.5 \pm 21.2$ & $95.7 \pm 56.4$ & .001 \\
ACP time, min & $100.8 \pm 25.5$ & $124 \pm 23.2$ & .013 \\
Circulatory arrest time, min & $17.2 \pm 4.2$ & $62.5 \pm 19.3$ & $<.001$ \\
Bladder temperature at initiation of & $30.1 \pm 0.7$ & $26.5 \pm 0.9$ & $<.001$ \\
circulation arrest, ${ }^{\circ} \mathrm{C}$ & $28.0 \pm 0.8$ & $24.9 \pm 0.7$ & .001 \\
Lowest bladder temperature, ${ }^{\circ} \mathrm{C}$ & $9.7 \pm 1.8$ & $18.3 \pm 4.6$ & .005 \\
Postoperative hospital stay, day & $8.6 \pm 1.4$ & $13.9 \pm 5.7$ & .015 \\
Intubation time, min & 0 & $3(4.6)$ & .699 \\
Stroke, $\mathrm{n}$ (\%) & 0 & 0 & \\
Paraplegia, $\mathrm{n}$ & 0 & 0 & \\
30-day morality, $\mathrm{n}$ & 0 & $8(12.5)$ & .37 \\
Acute kidney injury, $\mathrm{n}(\%)$ & & & \\
\hline
\end{tabular}

CPB indicates cardiopulmonary bypass; SCP, antegrade cerebral perfusion.

of developing AKI after TAR with moderate hypothermia $\left(24^{\circ} \mathrm{C}\right)$ has been reported to be $8.2 \%$ [Okita 2015]. Nota et al reported that mild hypothermic lower body circulatory arrest in TAR was associated with a low rate of kidney injury, but prolonged arrest time was an independent risk factor for AKI [Nota 2014]. In our study, AKI was not significantly different between the two groups, but we experienced zero cases of AKI in group B. This endo-balloon technique has a possibility of protecting renal function.

ACP performed under mild hypothermia may lower the risk of neurological complications and in-hospital mortality [Suzuki 2013], and further shortening of circulatory arrest time likely enhances neuroprotection. We report the use of an FET graft with endo-clamp to facilitate anastomosis at the distal aorta, achieving short circulatory arrest time while avoiding excessive systemic cooling. The FET graft consisted of a self-expanding stent anchored into a woven Dacron graft. It is used to perform anastomosis between the brachiocephalic artery and left common carotid artery, providing a good surgical window even in cases with a complex aneurysm. The benefit of this technique is that distal anastomosis is easily performed and has a shortened systemic cooling and rewarming time. Utilizing this method, we can minimize CPB and circulation arrest time, and reduce mechanical ventilation time and postoperative hospital stay. Prolonged mechanical ventilation after cardiovascular surgery is correlated with an increased risk of mortality [Kollef 1995]. Qian et al reported that prolonged CPB time was the stronger predictor with a 6-fold increased incidence of prolonged mechanical ventilation, and considered 180 minutes as an appropriate cutoff for prolonged CPB time [Lei 2009]. 
No patients developed neurological or renal complication in our series. Retrograde perfusion via the femoral artery and endo-clamp technique using FET successfully shortened the lower body circulatory arrest time, permitted mild hypothermia, and likely enhanced protection of the spinal cord and visceral organs. The excellent surgical results indicate the safety of mild hypothermia in the present study.

In conclusion, endo-clamp with FET graft can safely shorten circulation arrest time. This may enhance neural protection, avoiding coagulopathy and other adverse effects of hypothermia in TAR.

\section{REFERENCES}

Kato M, Ohnishi K, Kaneko M, et al. 1996. New graft implanting method for thoracic aortic aneurysm or dissection with a stented graft. Circulation 94: II188-93.

Kazui T, Washiyama N, Muhammad BA, et al. 2000. Total arch replacement using arch branched grafts with the aid of antegrade selective cerebral perfusion. Ann Thorac Surg 70:3-8.

Kollef MH, Wragge T, Pasque C. 1995. Determinants of mortality and multiorgan dysfunction in cardiac surgery patients requiring prolonged mechanical ventilation. Chest 107:1395-1401.

Lei Q, Chen L, Zhang Y, et al. 2009. Predictors of prolong mechanical ventilation after aortic arch surgery with deep hypothermic circulatory arrest plus antegrade selective cerebral perfusion. J Cardiothorac Vasc Anesth 23:495-500.

Nota T, Asai T, Suzuki T, et al. 2014. Risk factors for acute kidney injury in aortic arch surgery with selective cerebral perfusion and mild hypothermic lower body circulatory arrest. Interact Cardiovasc Thorac Surg 19:955-61.

Okita Y, Miyata H, Motomura N, et al. 2015. A study of brain protection during total arch replacement comparing Antegrade cerebral perfusion versus hypothermic circulatory arrest, with or without retrograde cerebral perfusion: analysis based on the Japan Adult cardiovascular surgery database. J Thorac Cardiovasc Surg 149:S65-73.

Preventza O, Coselli JS, Garcia A, et al. 2017. Moderate hypothermia at warmer temperatures is safe in elective proximal and total arch surgery: Results in 665 patients. J Thorac Cardiovasc Surg 153:1011-18.

Suzuki T, Asai T, Nota H, et al. 2013. Selective cerebral perfusion with mild hypothermic lower body circulatory arrest is safe for aortic arch surgery. Eur J Cardiothorac Surg 43:e94-98.

Uchino S, Bellomo R, Goldsmith D, et al. 2006. An assessment of the RIFLE criteria for acute renal failure in hospitalized patients. Crit Care Med 34:1913-17.

Yau TM, Carson S, Weisel RD, et al. 1992. The effect of warm heart surgery on postoperative bleeding. J Thorac Cardiovasc Surg 103:1155-63.

Zierer A, Detho F, Dzemali O, et al. 2011. Antegrade Cerebral perfusion with mild hypothermia for aortic arch replacement: single center experience in 245 consecutive patients. Ann Thorac Surg 91:1868-74. 